\title{
An advanced model for the short-term forecast of wind energy
}

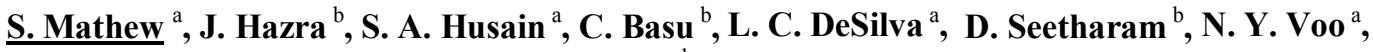 \\ S. Kalyanaraman ${ }^{\mathrm{b}}$ and Z. Sulaiman ${ }^{\mathrm{a}}$ \\ ${ }^{a}$ UBD $\mid$ IBM Centre, University of Brunei Darussalam, \\ Jalan Tungku Link, Gadong BE 1410, Brunei Darussalam \\ ${ }^{b}$ Next Gen Systems \& Smarter Planet Solutions Department \\ IBM Research - India, Nagawara, Bangalore - 560045, India \\ Email: sathyajith.mathew@ubd.edu.bn
}

\begin{abstract}
A novel short-term wind energy forecasting method, which is being developed under the University of Brunei Darussalam - International Business Machines (UBD-IBM) renewable energy modeling initiative, is described in this paper. The paper starts with a brief review on the existing forecasting methods. Prediction models based on the physical (derived from Numerical Weather_Prediction models) and Time Series approaches are discussed. The prediction errors under these methods are described and the need for a reliable forecasting system is emphasized. This is followed by a detailed discussion on the UBD-IBM approach. The baseline of the proposed forecasting system is the IBM Deep Thunder. Deep thunder is a highly modified version of the Regional Atmospheric Modeling System (RAMS). This high resolution Weather forecasting system can predict the local Weather variations on a 'day ahead' basis, at high accuracy levels. For a given wind farm site, specific Deep Thunder models could be developed and calibrated using the surface measured wind data. This enables the Deep Thunder to predict the wind profile at the wind farm locations over shorter time scales. Once the wind spectra at the reference height are obtained from the Deep Thunder, terrain characteristics of the wind farm site are introduced to the model using the geotropic drag law. This will further be extended to the specific turbine location by considering local variations in surface roughness, orographic effects etc. The wind farm wake effect is introduced to the model at this stage. Velocity deficit experienced by the downstream turbines due to the presence of the upstream ones would be modeled. A semi empirical approach, based on the conservation of momentum, would be adopted. With these procedures, the wind velocity, 'actually felt' by individual turbines in the farm could be predicted. From this predicted wind velocity at the hub height, the expected energy yield from individual turbines over a given time period is estimated. For this, the probability density of these wind speeds within the 'look-ahead period' and the power curve of the turbines are combined and integrated over the functional velocity range of the wind generator. The total energy expected from the wind farm can be computed by adding the energy yields from individual turbines.
\end{abstract}

Keywords: $\quad$ Wind Energy, Short-term Forecast, IBM Deep Thunder, Numerical Weather Prediction, Wake Effect, Weibull Distribution, Energy Model, Power Curve 


\section{INTRODUCTION}

Supplementing the energy base with renewable and sustainable energy resources has become imperative due to the present day's energy crisis and growing environmental concern. With a global installation of 194 GW and consistent annual growth rate over 25 percent over the past decade [1], wind is the most important renewable energy alternative in the world today. Due to this rapid growth in the wind power sector, the grid presence of wind energy has also increased significantly in the recent years. The growth of the wind power sector is expected to continue in the coming years as well. For example, projections by the Global Wind Energy Council (GWEC) indicate that, by the next 20 years, the cumulative wind power capacity would reach between $572 \mathrm{GW}$ and 2,341GW, under various policy scenarios [2]. Thus, under favorable conditions, the share of wind energy in the global power generation could reach up to 22 percent, making wind the major player in the global energy market, meeting a fifth of the world's power demand.

One of the critical problems in the large scale integration of the wind generated electricity with the grid is the uncertainty in its availability due to the stochastic nature of wind. An estimate on the available wind energy over shorter time scales (0-48 hours) is essential for the successful integration of wind power systems with the grids, from where it is being dispatched along with the electricity generated from other conventional options. Such a forecast is critical for the utilities for ensuring the unit commitment, economizing the dispatch, formulating the tariff structures and assessing the dynamic security [3]. Technical and economical advantages of such forecasting in a dynamic electricity market is well established [4,5]. Though several attempts have already been made for the short-tem forecast of wind power, the accuracy level of these prediction systems is not very impressive $[6,7,8]$. In this paper we present the structure of a novel forecasting approach which is being developed under the UBD-IBM renewable energy modeling initiative. Some preliminary model results are also included.

\section{METHODS FOR SHORT-TERM FORECASTING}

Two different approaches are adopted for forecasting the wind power availability over shorter time periods. They are: (1) Physical approach and (2) Statistical time series approach. The physical approach is based on the Numerical Weather Prediction (NWP) models. Characteristics of the expected wind profile over a larger area containing the wind farm would be generated by the NWPs. This is further extrapolated to the surface wind at the hub height of the turbines by incorporating the local conditions like surface roughness and orographic effect at the wind farm location. Once the surface winds expected over the farm is known, the power outputs of the turbines are calculated using the power curve of the wind turbine. One of such NWP models, commonly used for wind power forecast, is the High Resolution Limited Area Model (HiRLAM) [3].

On the other hand, the statistical time series approach is based on the relationship between the historical values of meteorological variables and wind power production. The relationship thus developed could also be regularly modified with meteorological forecasts and power measurements during the online operation of the prediction system. Apart from different linear and non-linear statistical models, artificial-intelligence-based black-box models like Neural-Networks (NNs) [9,10] and Support Vector Machines (SVM) [11] are also being used for time series based wind power prediction.

The major problem with both these short term wind power forecasting methods is the low accuracy level. The accuracy depends on several factors like the model characteristics, prediction length and local wind farm conditions. For example, a wide variation (10-45 percent) in the normalized absolute average prediction error is reported under certain studies [6].

\section{THE UBD-IBM APPROACH}

Based on the previous investigations, an advanced short term wind power forecast model is being proposed under the UBD-IBM renewable energy modeling programme. Salient features of the proposed approach are:

- Higher resolution NWP model for defining the wind profile

- Improved wake model for characterizing the wake effect within the wind farm

- Using direction dependant roughness and orographic models

- Improved energy model which incorporates the variations in wind velocity within the forecasting period using statistical distributions

- Power curve based on wind farm measurement instead of using the theoretical power curve provided by the turbine manufacturers 


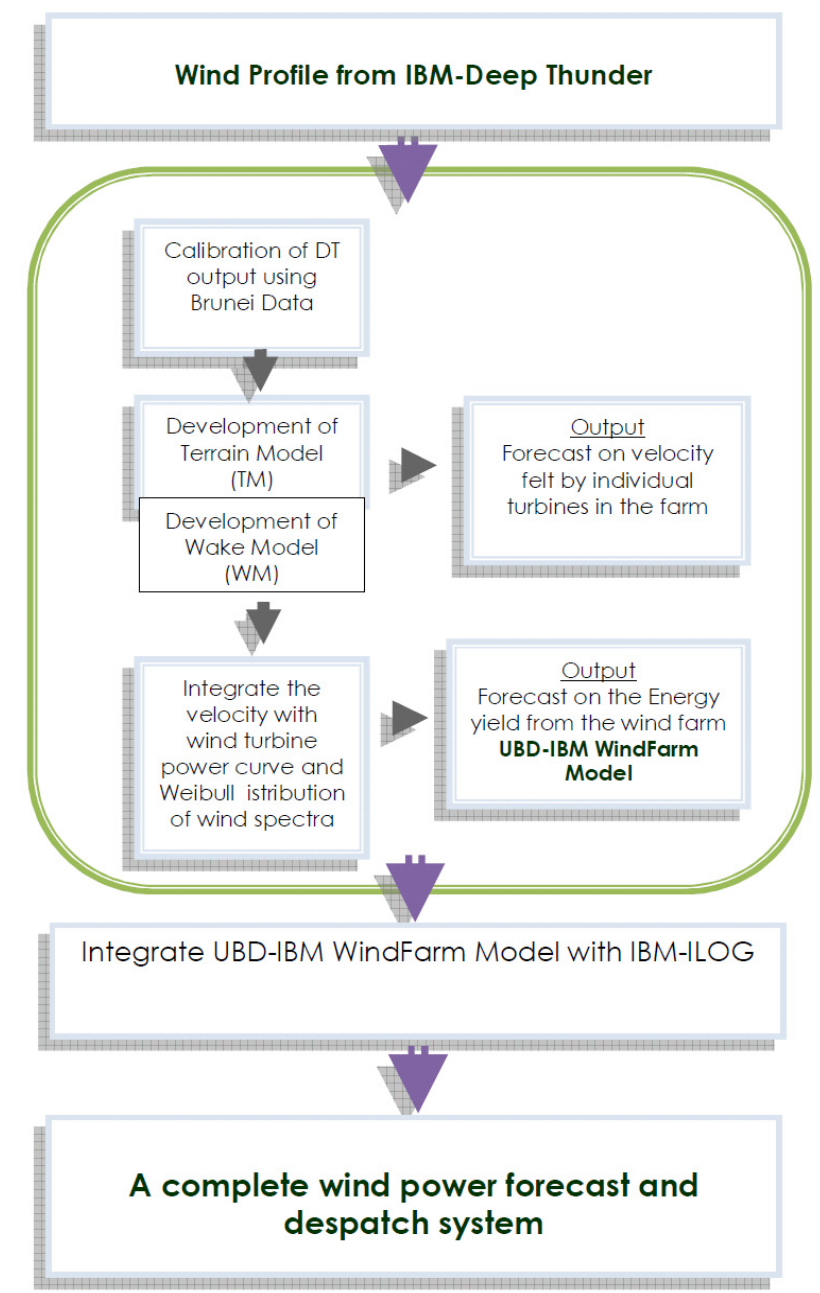

Figure 1. The UBD-IBM short term wind energy forecast method

Schematic representation of the UBD-IBM short term wind energy forecast model is shown in Fig. 1. The baseline of the proposed forecasting system is the IBM Deep Thunder. This high resolution Weather forecasting system can predict the local Weather variations on a 'day ahead' basis, at high accuracy levels. For a given wind farm site, specific Deep Thunder models could be developed and calibrated using the surface measured wind data. This enables the Deep Thunder to predict the wind profile at the wind farm locations over shorter time scales.

Once the wind spectra at the reference height are obtained from the Deep Thunder, terrain characteristics of the wind farm site are introduced to the model using the geotropic drag law. This will further be extended to the specific turbine location by considering local variations in surface roughness, orographic effects etc. The wind farm wake effect would be introduced to the model at this stage. Velocity deficit experienced by the downstream turbines due to the presence of the upstream ones would be modeled. A semi empirical approach, based on the conservation of momentum, would be adopted.

With these procedures, the wind velocity, 'actually felt' by individual turbines in the farm could be predicted. From this predicted wind velocity at the hub height, the expected energy yield from individual turbines over a given time period is estimated. For this, the probability density of these wind speeds within the 'look-ahead period' and the power curve of the turbines are combined and integrated over the functional velocity range of the wind generator. The total energy expected from the wind farm can be computed by adding the energy yields from individual turbines.

The project is under progress and some of the preliminary results are presented in the following sections. 


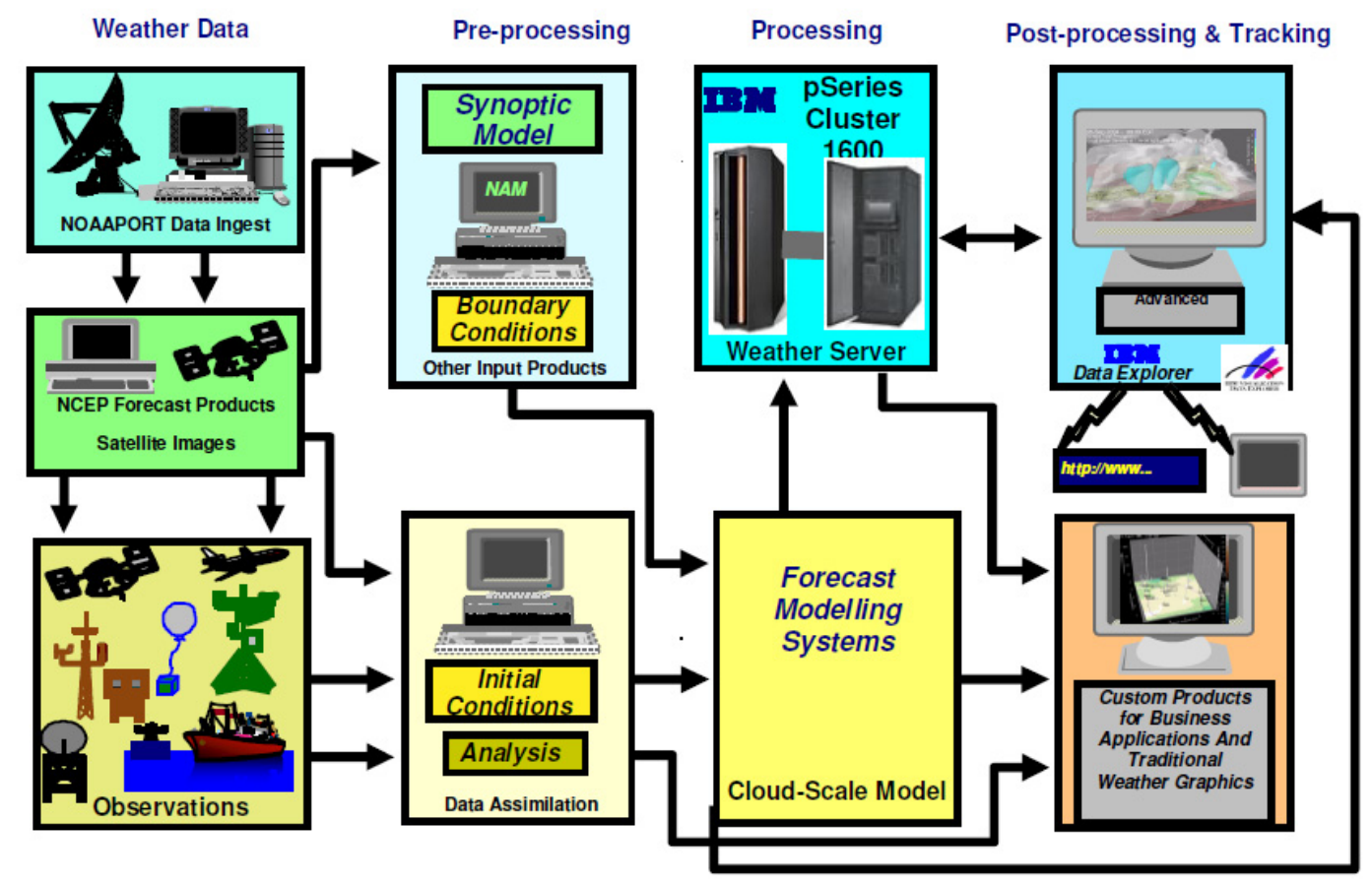

Figure 2. Deep Thunder architecture

\section{THE DEEP THUNDER}

The architectural features of Deep Thunder are shown in Fig. 2. Deep Thunder is a service that provides local, high-resolution weather predictions customized to business applications for weather-sensitive operations up to a day ahead of time. In particular, the goal is to provide weather forecasts at a level of precision and fast enough to address specific business problems. Deep Thunder produces forecasts that provide detailed four-dimensional information about temperature, winds, precipitation, etc. from the surface of the earth to an altitude of about $15 \mathrm{~km}$. The focus of DT is on high-performance computing, visualization, and automation while designing, evaluating and optimizing an integrated system that includes receiving and processing data, modeling, and post-processing analysis and dissemination. Rapid computation is insufficient if the results cannot be easily and quickly utilized. Thus, a variety of fixed and highly interactive flexible visualizations focused on the applications have also been implemented to enable timely use and assessment of the model forecasts. The model configuration includes full bulk cloud microphysics (e.g., liquid and ice) for three nests to enable explicit weather prediction. Each nest employs specific time step (e.g. 48, 12 and 3 seconds) to ensure computational stability and to also accommodate strong vertical motions that can occur during modeling of severe convection. Each nest employs the same vertical grid using 31 stretched levels with the lowest level at $48 \mathrm{~m}$ above the ground, a minimum vertical grid spacing of $100 \mathrm{~m}$, a stretch factor of 1.12 and a maximum grid spacing of $1000 \mathrm{~m}$. The data for both boundary and initial conditions for each model execution is derived from several sources like Numerical Weather Prediction, satellite image, etc. The static and dynamic data are processed via an isentropic analysis package to generate three-dimensional data on the model nested grids for direct utilization.

DT consists of 4 modules i.e. data collection, pre-processing, processing and post-processing as shown in Fig. 2. Each module is described below.

\subsection{Data}

The NOAAport system provides a number of different data sources as disseminated by the NWS. These include in site and remotely sensed observations for model boundary and initial conditions. The various files transmitted via NOAAport are converted into conventional files in their native format and used for preprocessing. 


\subsection{Pre-Processing}

The pre-processing consists of two parts. The first is essentially a parsing of the data received via NOAAport into usable formats whereas the second part consists of analysis and visualization. Specialized processing and analysis has been implemented to assure quality control and appropriate utilization of these data in the module pre-processing.

\subsection{Processing}

To enable timely execution of the forecast modules, which is required for operations, the simulation is parallelized on a high-performance computing system. For this effort, an IBM RS/6000 Scalable Power Parallel (SP) and an IBM pSeries Cluster 1600 are employed.

\subsection{Post-Processing}

Post-processing essentially operates on the raw model output. There are several aspects of post-processing, the most important of which is visualization. Since large volumes of data are produced, which are used for a number of applications, the parallel computing system is used for post-processing.

\section{MODELING THE WIND FARM WAKES}

When the wind stream interacts with a turbine, the flow is disturbed due to the rotation of the blades and a wake is left behind. As the flow proceeds downstream, these wakes get diluted due to spreading and the flow would regain the free stream conditions after a certain distance. In a wind farm, where a number of turbines are clustered, the wind speed experienced by a downstream turbine will be lesser than that received by the upstream turbine due to this wake effect. Hence, the local wind velocity, generated by the Deep Thunder, has to be corrected for the wake effect for truly representing the wind flow within the wind farm. An improved $3 \mathrm{D}$ wake model is proposed under the project, considering the positions of the turbine, direction of wind flow and variations in hub heights of the turbines.

Based on the simple wake model suggested by Katic et.al. [12], the wind speed at any downstream turbine at a distance $\mathrm{x}$ from the upstream turbine can be written as:

$$
V=U\left[1-\left(1-\sqrt{1-C_{T}}\right)\left(\frac{D}{D+2 K x}\right)^{2}\right]
$$

where, $\mathrm{U}$ is the free wind speed, $\mathrm{V}$ is wake wind speed, $C_{T}$ is the thrust coefficient of the turbine; $\mathrm{K}$ is the wake decay constant; $\mathrm{x}$ is the horizontal distance behind the upstream turbine and $\mathrm{D}$ is the rotor diameter of the upstream turbine.

The above formula will be valid only if the wake of the upstream turbine covers the full swept area of the downstream turbine. In practice, wake of the upstream turbine may intersect a portion of the swept area of the downstream turbine either because of wind direction or because of different hub heights. Let $A_{\text {overlap }}$ denotes the swept area of the downstream turbine subjected to the wake effect caused by the upstream turbine. Let $f_{\text {overlap }}=\frac{4 \times A_{\text {overlap }}}{\Pi D^{2}}$ be the corresponding fraction of area. Then we have the following expression.

$$
V=U\left[1-\left(1-\sqrt{1-C_{T}}\right)\left(\frac{D}{D+2 K x}\right)^{2} \times f_{\text {overlap }}\right]
$$

or,

$$
V=U[1-d]
$$

where $\mathrm{d}$ is usually referred as depression coefficient, given by:

$$
d=\left(1-\sqrt{1-C_{T}}\right)\left(\frac{D}{D+2 K x}\right)^{2} \times f_{\text {overlap }}
$$



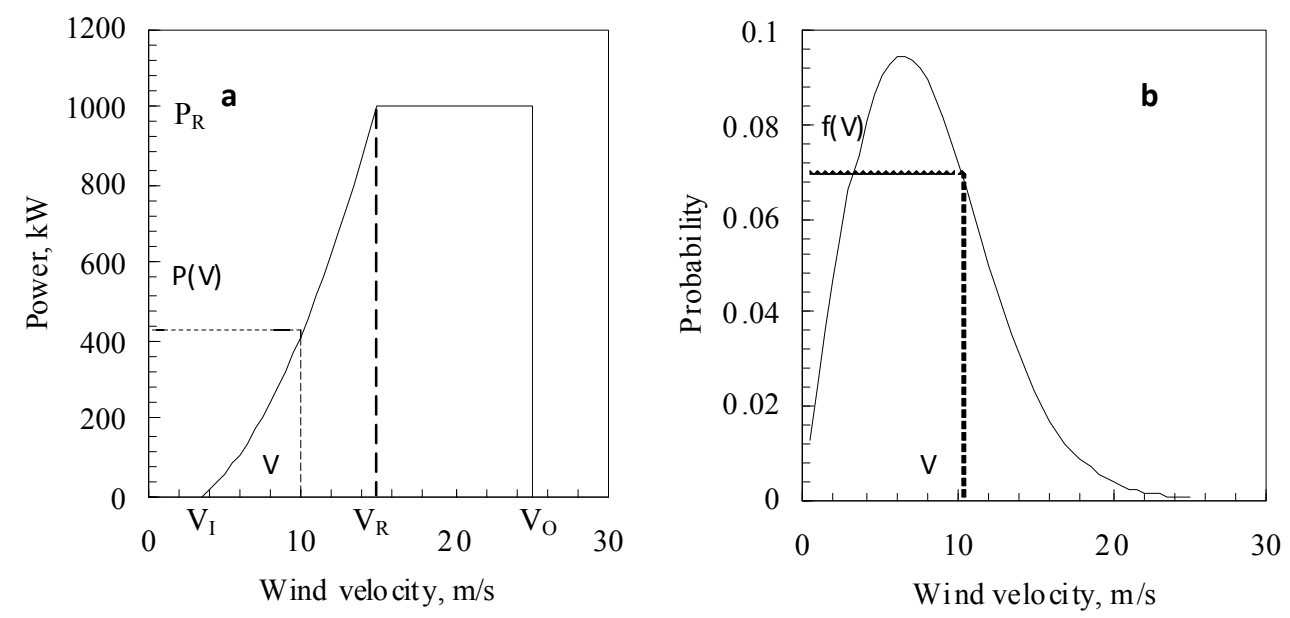

Figure. 3 Power curve of the wind turbine (a) and wind velocity distribution (b)

If the upstream turbine is placed at a height of $h_{1}$ and the downstream turbine is placed at a height of $h_{2}$, then the velocity expression can be written as follows:

$$
V=U_{h 2}\left[1-d\left(\frac{U_{h 1}}{U_{h 2}}\right)^{2}\right]
$$

where $U_{h 1}$ and $U_{h 2}$ are the upwind velocities at height $h_{1}$ and $h_{2}$ respectively. The relationship of wind velocity with height can be written as:

$$
\frac{U_{h 1}}{U_{h 2}}=\left[\frac{h_{1}}{h_{2}}\right]^{\alpha}
$$

where $\alpha$ is the coefficient of surface roughness. Substituting above relation in the velocity expression we get:

$$
V=U_{h 2}\left[1-d\left(\frac{h_{1}}{h_{2}}\right)^{2 \alpha}\right]
$$

In general term, the wake wind speed at turbine $n$ due to wake of turbine $i$ can be written as:

$$
V_{i, n}=U_{n}\left[1-d\left(\frac{h_{i}}{h_{n}}\right)^{2 \alpha}\right]
$$

Due to multiple wakes, wind speed of any downstream turbine $n$ can be written as:

$$
V_{n}=U_{n}-\sqrt{\sum_{i=1 ; i \neq n}\left(U_{n}-V_{i, n}\right)^{2}}
$$

\section{THE ENERGY MODEL}

A typical power curve of a wind turbine is shown in Fig. 3 (a). As we can see, there are two productive regions in the curve. (1) Between the cut-in and rated velocities $\left(V_{I}\right.$ to $\left.V_{R}\right)$, where the power increases with the wind velocity. Though, theoretically, this increase should be cubic in nature, in practice it can be linear, quadratic, cubic and even higher powers and its combinations, depending upon the design of the turbine. (2) From the rated to cut-out wind speed $\left(V_{R}\right.$ to $\left.V_{O}\right)$, the power is constant at the rated power $P_{R}$, irrespective of 
the change in wind velocity. The power developed by the turbine $\mathrm{P}(\mathrm{V})$ at any wind velocity $\mathrm{V}$ in the first region can be given by $[13,14]$

$$
P_{V}=P_{R}\left(\frac{V^{n}-V_{I}^{n}}{V_{R}^{n}-V_{I}^{n}}\right)
$$

where $P_{R}$ is the rated power, $V_{I}$ is the cut-in velocity and $V_{R}$ is the rated velocity. The velocity-power proportionality $\mathrm{n}$ for any particular turbine can be computed by fitting this expression with the measured performance data.

In order to take care of the variations in the wind velocity within the forecast period, probability distribution of wind velocities (Fig. 3 (b)) is introduced in the model. Weibull distribution is considered in the current approach as it is well accepted for wind energy calculations [15]. With the above velocity-power relationship and probability distribution of wind velocities, the energy contributed by the first productive region $\left(\mathrm{E}_{\mathrm{IR}}\right)$ can be computed by

$$
E_{I R}=T \int_{V_{I}}^{V_{R}} P_{V} f(V) d V
$$

where $\mathrm{T}$ is the forecast time and $f(\mathrm{~V})$ is the probability distribution function. For Weibull distribution, probability distribution is given by

$$
f(V)=\frac{k}{c}\left(\frac{V}{c}\right)^{k-1} e^{-(V / c)^{k}}
$$

where $\mathrm{k}$ and $\mathrm{c}$ are the Weibull shape factor and scale factor respectively. Similarly, the energy contributed by the second productive region $\left(\mathrm{E}_{\mathrm{RO}}\right)$ is given by:

$$
E_{R O}=T P_{R} \int_{V_{R}}^{V_{O}} f(V) d V
$$

where $\mathrm{V}_{\mathrm{o}}$ is the cut-out wind velocity. Considering $\mathrm{n}$ turbines in the wind farm, the total energy yield from the farm over the forecasting period $\left(\mathrm{E}_{\mathrm{Farm}, \mathrm{T}}\right)$ is given by

$$
\mathrm{E}_{\mathrm{Farm}, \mathrm{T}}=\sum_{i=1}^{j}\left(E_{I R}+E_{R O}\right)
$$

\section{CONCLUSIONS}

Preliminary results of the UBD-IBM short-term wind energy forecast modeling project is presented in this paper. Existing modeling approaches are briefly outlined and the salient features of the UBD-IBM approach are highlighted. Initial formulations of the wake and energy models are also presented. With the high resolution weather prediction system like IBM Deep Thunder as its base and incorporation of advanced 3D wake and energy models, the new approach is expected to show better performance in short-term wind energy modeling. The work is in the initial stage and the wake and energy models are to be fine tuned and validated with the field observations. Further, a platform to accommodate the Deep Thunder output and different model algorithms are to be developed.

\section{ACKNOWLEDGMENTS}

The authors acknowledge the support provided by the UBD-IBM research centre for conducting this research. 


\section{REFERENCES}

[1] GWEC ( 2011). Global wind report 2010. Global Wind Energy Council, Rue d'Arlon 801040 Brussels, Belgium

[2] GWEC (2011). Global Wind Energy Outlook 2010, Global Wind Energy Council, Rue d'Arlon 80 1040 Brussels, Belgium

[3] Giebel, G., Brownsword, R. and Kariniotakis, G. (2003). The State-Of-The-Art in Short-Term Prediction of Wind Power: A Literature Overview. Project Report, ANEMOS, funded by the European Commission, No. ENK5-CT-2002-00665.

[4] Barthelmie, R.J., Murray, F., and Pryor S.C. (2008). The economic benefit of short-term forecasting for wind energy in the UK electricity market. Energy Policy 36 : 1687-1696

[5] Boqiang, R and Chuanwen, J (2009). A review on the economic dispatch and risk management considering wind power in the power market. Renewable and Sustainable Energy Reviews,13(8), 2169-2174.

[6] Maria Grazia, G., Ficarella, A. and Tarantino, M (2011). Error analysis of short term wind power prediction models. Applied Energy, 88 , 1298-1311.

[7] Neilson, H, A. (2011). Short term Forecast of Wind Power. In Mathew, S and Philip, G.S. (Eds), Advances of Wind Energy Conversion Technology, Springer.

[8] Focken, U., Lange, M., Onnich, K.M., Waldla, H.P., Beyerb, H. G. and Luigb, A. (2002). Shortterm prediction of the aggregated power output of wind farmsFa statistical analysis of the reduction of the prediction error by spatial smoothing effects. Journal of Wind Engineering and Industrial Aerodynamics, 90, 231-246.

[9] Foley, A.M., Leahy, P.G., Marvuglia, A. and McKeogh, E.J. (2011). Current methods and advances in forecasting of wind power generation. Renewable Energy xxx (2011) 1-8

[10]Lei, M., Shiyan, L., Chuanwen., J, Hongling, L. and Yan, Z. (2009). A review on the forecasting of wind speed and generated power. Renewable and Sustainable Energy Reviews 13, 915-920.

[11] Mohandes, M.A., Halawani, T. O., Rehman, S. and Ahmed A. Hussain (2004). Support vector machines for wind speed prediction. Renewable Energy, 29(6), 939-947

[12] Katic, I., Højstrup, J. and Jensen, N.O. (1986). A Simple Model for Cluster Effeciency. European Wind Energy Association Conference and Exhibition, 7-9 October 1986, Rome, Italy.

[13] Mathew , S. (2006) Wind Energy: Fundamentals, Resource Analysis and economics, first ed., Springer-Verlag, Berlin Heidelberg.

[14] Mathew , S. and Philip, G.S. (2011). Advances in Wind Energy Conversion Technology, first ed., Springer-Verlag, Berlin Heidelberg.

[15] Mathew, S., Pandey, K.P. and Anilkumar V. (2002). Analysis of wind regimes for energy estimation. Renewable Energy, Vol. 25 pp 381-399. 